ARTICLE

\title{
Genome-wide analysis of the human p53 transcriptional network unveils a IncRNA tumour suppressor signature
} Yolanda Sánchez ${ }^{1}$, Victor Segura ${ }^{1}$, Oskar Marín-Béjar ${ }^{1, \star}$, Alejandro Athie ${ }^{1, \star}$, Francesco P. Marchese $^{1, \star}$,
Jovanna González ${ }^{1}$, Luis Bujanda ${ }^{2}$, Shuling Guo ${ }^{3}$, Ander Matheu $^{4,5} \&$ Maite Huarte $^{1}$

Despite the inarguable relevance of p53 in cancer, genome-wide studies relating endogenous p53 activity to the expression of IncRNAs in human cells are still missing. Here, by integrating RNA-seq with p53 ChIP-seq analyses of a human cancer cell line under DNA damage, we define a high-confidence set of 18 IncRNAs that are p53 transcriptional targets. We demonstrate that two of the p53-regulated IncRNAs are required for the efficient binding of p53 to some of its target genes, modulating the p53 transcriptional network and contributing to apoptosis induction by DNA damage. We also show that the expression of p53-IncRNAs is lowered in colorectal cancer samples, constituting a tumour suppressor signature with high diagnostic power. Thus, p53-regulated IncRNAs establish a positive regulatory feedback loop that enhances p53 tumour suppressor activity. Furthermore, the signature defined by p53-regulated IncRNAs supports their potential use in the clinic as biomarkers and therapeutic targets.

\footnotetext{
${ }^{1}$ Center for Applied Medical Research, University of Navarra, 55 Pio XII Avenue, 31008 Pamplona, Spain. ${ }^{2}$ Department of Gastroenterology, Donostia Hospital-Biodonostia Research Institute, University of Basque Country (UPV/EHU), Biomedical Research Center in the Network of Digestive and Hepatic Diseases (CIBERehd), Dr Begiristain Square, 20014 San Sebastian, Spain. ${ }^{3}$ Department of Antisense Drug Discovery, Isis Pharmaceuticals, 2855 Gazelle Court, Carlsbad, California 92008, USA. ${ }^{4}$ Neuro-Oncology Section, Oncology Department, Biodonostia Research Institute, Dr Begiristain Square, 20014 San Sebastian, Spain. ${ }^{5}$ Ikerbasque, Basque Foundation for Science, Bilbao 48013, Spain. ${ }^{\star}$ These authors contributed equally to this work. Correspondence and requests for materials should be addressed to M.H. (email: maitehuarte@unav.es).
} 
T he transcription factor p53 is the most prominent human tumour suppressor. p53 is essential for the cellular response to DNA-damaging stimuli to maintain genomic integrity of cells, mainly by activating a gene expression programme that leads to cell cycle arrest or elimination of the damaged cells through programmed cell death. The vast majority of the p53 downstream effects are mediated through its intrinsic nature as transcription factor ${ }^{1}$. On cellular stress, p53 protein is stabilized and can recognize its target genes through binding to a consensus response element (p53RE) located proximal to the transcription start site (TSS) at the gene promoter, the first intron or even further downstream of the gene ${ }^{2}$. For decades, researchers have focused their attention on the protein-coding genes regulated by $\mathrm{p} 53$, which led to the discovery of a large set of proteins involved in the p53 response. However, recent progress has suggested that a significant number of p53REs lie on noncoding regions of the genome and that some of these genomic loci are transcribed into long noncoding RNAs (lncRNAs).

LncRNAs are transcripts longer than 200 nucleotides that lack functional open reading frames ${ }^{3,4}$. Similarly to mRNAs, lncRNAs are frequently polyadenylated and spliced, and their promoters are subjected to regulation by transcription factors such as $\mathrm{p} 53$. Distinctive features of IncRNAs are their highly specific expression patterns and relatively low conservation across species, consistent with their role as regulatory molecules that fine-tune gene expression ${ }^{4-7}$.

Few IncRNAs have been studied in some depth. These show the important roles of lncRNAs in many processes that involve gene regulation, such as cellular differentiation, proliferation, dosage compensation and chromosomal imprinting ${ }^{3,8-12}$. Given their physiological activities, the deregulation of lncRNAs is one of the underlying causes of human disease, including cancer, and they emerge as promising targets for novel therapies ${ }^{13-15}$.

Our work and others' have shown that p53 regulates the expression of some lncRNAs. For instance, we identified lincRNA-p21 (ref. 16) and Pint ${ }^{17}$, which modulate cellular apoptosis and proliferation in mouse cells. Studies by other groups lead to the identification of $P A N D A^{18}$, a lncRNA able to inhibit cellular apoptosis in human fibroblasts, lincRNA-RoR and loc285194 (refs 19,20), reported as post-tanscriptional regulators in the p53 pathway, and TUG-1, which controls proliferation in human non-small cell lung cancer ${ }^{4,21}$.

Although these studies underscore a functional role of lncRNAs in the p53 pathway, the extent of the contribution of lncRNAs to the p53 response to DNA damage in human cells still remains poorly understood.

In this study, we integrate genome-wide expression data obtained by RNA sequencing (RNA-seq) with p53 ChIP-seq data of human cancer cells treated or not with a DNA-damaging drug. The combination of these experimental approaches allowed us to relate the active binding of $\mathrm{p} 53$ to the expression of protein-coding and -noncoding regions of the genome, including regions poorly or not annotated previously. We also provide experimental evidence of the contribution of a subset of lncRNAs to the p53 human transcriptional network and biological activity. Finally, we show a tumour suppressor signature defined by p53-regulated lncRNAs.

\section{Results}

Hundreds of IncRNAs are affected by DNA-damage treatment in human cells. We set out to investigate the polyadenylated human transcriptome regulated by $\mathrm{p} 53$. To that end, we used as a model the HCT116 colorectal cancer cell line, which has been previously reported to have an intact $\mathrm{p} 53$ response $\mathrm{e}^{22}$ (Supplementary Fig. 1a). To induce p53 protein stabilization and transcription of its target genes, cells were treated with the
DNA-damage-inducing drug 5-fluorouracil (5-FU) for different times $(0,4$ and $12 \mathrm{~h})$. We then isolated the polyadenylated RNA fraction and performed strand-specific paired-end RNA Illumina sequencing with reads of 150 base pairs (bp) length (Supplementary Fig. 1b). On average, we obtained 170M mapped reads per experimental condition, which were assembled using Cufflinks and Cuffmerge ${ }^{23}$ (See Methods and Suplementary Methods). In total, 131,936 transcripts were successfully assembled, of which $85 \%$ were annotated according to Gencode v19 (refs 24,25). Out of the annotated transcripts, $76 \%(85,865)$ were identified as protein-coding mRNAs, while the remaining $24 \%$ were classified as different types of noncoding transcripts. A total of $14 \%(15,776)$ were defined as lncRNAs, including antisense, intergenic, processed transcripts, senseoverlapping and sense-intronic lncRNAs, while the remaining $10 \%(6,825)$ of annotated transcripts corresponded to other types of noncoding RNAs, such as transcripts derived from pseudogenes, retained introns and pri-microRNAs (Fig. 1a). This relative distribution of transcripts is similar to that described by Encode $^{25}$. We also found a large number of unassigned transcripts $(20,092)$, many of which were unspliced and which could partially be an artefact caused by incomplete determination of the transcript structures. To address this point, we analysed publicly available ChIP-seq data from HCT116 cells ${ }^{26}$. While the genomic region ranging from $-5 \mathrm{~kb}$ to $+5 \mathrm{~kb}$ around the $5^{\prime}$ end of $83 \%$ of the unassigned transcripts defined by our RNA-seq was not associated with an active transcription chromatin mark (that is, $\mathrm{H} 3 \mathrm{~K} 4 \mathrm{me} 3, \mathrm{H} 3 \mathrm{~K} 4 \mathrm{mel}$ or H3K27Ac; Fig. 1c), the remaining $17 \%$ were enriched by at least one of these histone marks at their $5^{\prime}$ end, suggesting the presence of a promoter driving their expression.

Next, to determine what transcripts are perturbed by the DNA-damage treatment, we applied Cuffdiff 2 (ref. 27; see Methods). Comparing HCT116 cells treated with DNA damage for $12 \mathrm{~h}$ to untreated cells, 4,050 transcripts were found differentially expressed $(P<0.01$, Cuffdiff differential expression analysis based on the beta-negative binomial distribution) in at least one of the two replicates. A total of 1,738 of these RNAs were upregulated, while 2,313 were downregulated upon drug treatment. Out of the total number of transcripts differentially expressed, 60\% corresponded to protein-coding genes (Fig. 1b; Supplementary Table 1) and, as expected, included well-known canonical p53 target genes such as CDKN1A, BBC3, PCNA and $B A X$ (Fig. 1d; Supplementary Table 1). To obtain a global view of the efficacy of the DNA-damage treatment as well as the activation of the p53 response, the whole set of protein-coding genes differentially expressed in our RNA-seq was used to predict upstream regulators and cellular pathways in silico. As expected, the most significant upstream regulator predicted was the transcription factor p53 $(P=6.04 \mathrm{E}-37$, Ingenuity Fisher's exact test; Supplementary Fig. 1c) and the most enriched canonical pathway was the p53 signalling, followed by ataxia-telangiectasia mutated (ATM) signalling pathway (Supplementary Fig. 1d). These results confirmed that the genes selected by our analysis are enriched in those of the p53 transcriptional response to DNA damage.

Besides the protein-coding transcripts, we aimed to identify lncRNAs differentially expressed following DNA damage. A total of 1,531 RNAs with expression values significantly affected by the DNA-damage treatment were found (Supplementary Table 1). A total of 633 corresponded to lncRNAs, distributed as intergenic (190), antisense (122), sense-overlapping lncRNAs (19), processed transcripts (80) and transcripts derived from pseudogenes (232; Fig. 1e). In addition, we found 888 unassigned transcripts affected by the drug treatment that, as discussed above, may account in part for artefacts due to technical limitations associated with RNA-seq data production and analysis. 
a

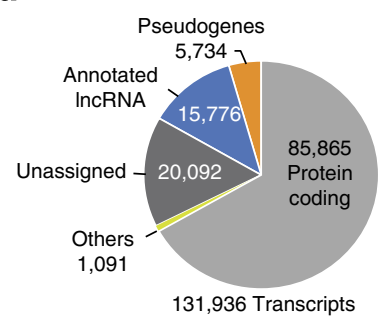

b

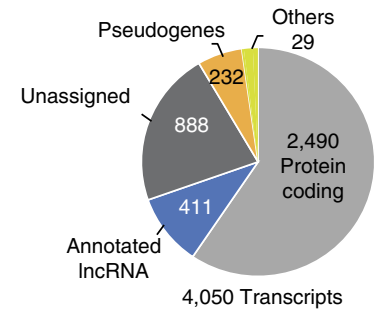

c

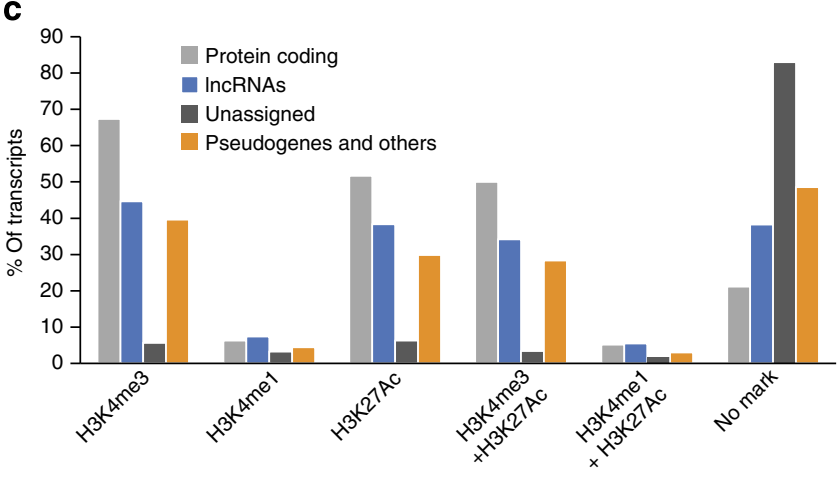

d
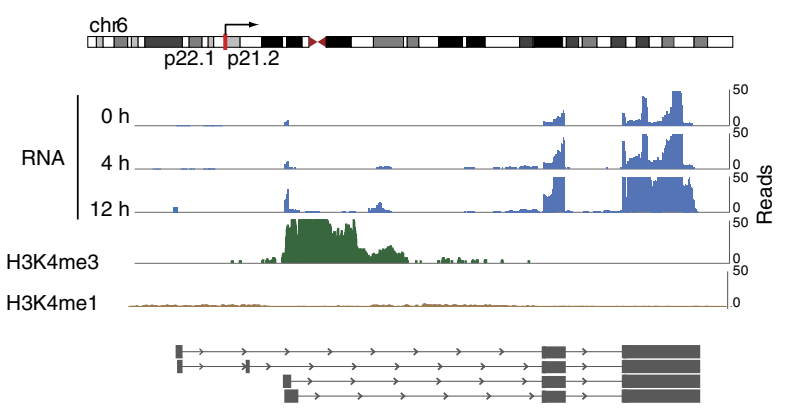

CDKN1A

e

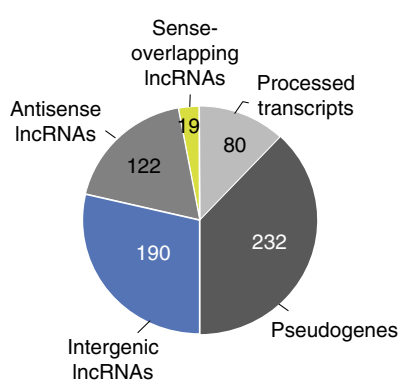

Figure 1 | Genome-wide analysis of the DNA-damage transcriptome of HCT116 cells. (a) Biotype distribution of all the transcripts identified by RNA-seq analysis generated from HCT116 cells untreated or treated with DNA damage annotated relatively to ENCODE v19. (b) Biotype distribution of the differentially expressed transcripts following DNA-damage treatment with 5-FU for $12 \mathrm{~h}$. (c) Percentage of the transcripts identified by RNA-seq with $\mathrm{H} 3 \mathrm{~K} 4 \mathrm{me} 3, \mathrm{H} 3 \mathrm{~K} 4 \mathrm{me} 1$ or/and H3K27ac marks at the $5^{\prime}$ position of the defined transcript structures. (d) Schematic representation of the chromosomal location of the CDKN1A gene locus, RNA expression detected by RNA-seq of DNA-damage-treated cells and, H3K4me3 and H3K4me1 levels of HCT116 untreated cells and CDKN1A transcript isoforms as assembled by Cufflinks. (e) Subtypes' distribution of the IncRNAs found differentially expressed following 5-FU treatment.

To validate our results, we performed quantitative reverse transcription PCR (qRT-PCR) for a selection of 26 differentially expressed lncRNAs on HCT116 cells treated with DNA damage (23 upregulated and 3 downregulated on DNA damage; Supplementary Fig. 2) and confirmed the differential expression for 23 (20 up- and 3 downregulated) out of the 26 lncRNAs (88\%).

In summary, we compiled a genome-wide catalogue of proteincoding and noncoding transcripts affected or not by DNA damage in HCT116 cells and identified a set of lncRNAs that are differentially expressed upon DNA damage in human cells.

p53 associates to multiple lncRNA gene loci. Our gene expression analysis identified transcripts with altered expression upon DNA damage, which comprises genes of the p53 response. However, it was unable to distinguish those directly regulated by $\mathrm{p} 53$.

To discern p53 direct targets, we performed p53 ChIP-Seq on untreated HCT116 cells as well as cells treated with DNA damage (12 h of 5-FU treatment). ChIP-seq analysis identified a total of 3,617 p53 peaks in the DNA-damage-treated cells (Fig. 2a). Interestingly, p53 was bound to 1,481 sites even in the absence of DNA damage, indicating the p53 basal activity in the cells. In many cases, the peaks detected even in the absence of treatment corresponded to known bona fide p53 target genes such as $C D K N 1 A, B A X$ and $B B C 3$ (Supplementary Table 2). However, the intensity of p53 binding at these loci, as well as the number of peaks and average global signal intensity, was increased upon DNA-damage treatment (Fig. 2a-c,e; Supplementary Fig. 3a-d).

The p53 ChIP-seq peaks were annotated relative to Gencode v19 and to our RNA-seq analysis in the case of the novel transcripts not previously annotated. A total of 582 gene loci were bound by $\mathrm{p} 53$, of which 260 are protein-coding genes, 80 lncRNAs and 155 unassigned genomic regions (Fig. 2d). Analysis of the relative position of the p53-binding peaks showed that $60 \%$ of them localized within $10 \mathrm{~kb}$ from the TSS of the nearest gene (proximal peaks), while the remaining $40 \%$ lied more than $10 \mathrm{~kb}$ apart (distal peaks). Interestingly, in the case of protein-coding genes, the positional distribution of the p53 proximal peaks was relatively uniform within the $20 \mathrm{~kb}$ window (Fig. 2e), while the majority of the proximal p53 peaks associated with lncRNAs were concentrated less than $5 \mathrm{~kb}$ downstream of the TSS (Fig. 2e). On the other hand, the analysis of the signal distribution showed that the ChIP-seq signal was enriched around the TSS of genes and also at $5 \mathrm{~kb}$ upstream of the TSS (Fig. 2f). This pattern of distribution of p53 signal was common to all gene types, independently of their coding or noncoding nature, indicating that the most intense $\mathrm{p} 53$ binding occurs around the 0 and $-5 \mathrm{~kb}$ positions.

p53 regulates the expression of lncRNAs upon DNA damage. Next, to determine the effect of p53 binding on gene expression, we compared the p53 ChIP-seq data with the RNA-seq analysis. In DNA-damage-treated cells, p53 was found bound to 109 genes that also showed at least one transcript differentially expressed when only considering the p53 proximal peaks $(<10 \mathrm{~kb}$ of distance to TSS; Fig. 2e). A total of $75 \%$ of these p53 directly regulated genes were upregulated on DNA damage, while only $25 \%$ were downregulated (Supplementary Table 3). To confirm the presence of the p53-binding motifs, we performed de novo motif analysis using the genomic sequences associated with the p53-binding peaks of those transcripts differentially expressed (see Methods). In agreement with the binding of p53, the p53 consensus motif was found highly enriched across the p53-bound loci (motif similarity $P=2.12 \mathrm{E}-10$, TOMTOM match statistic; Fig. 2g), confirming the presence of p53REs in these genomic regions. 
a

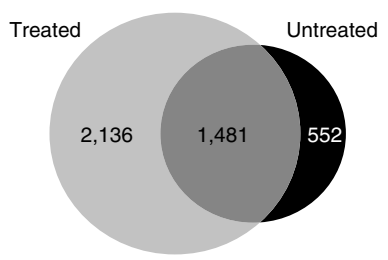

b
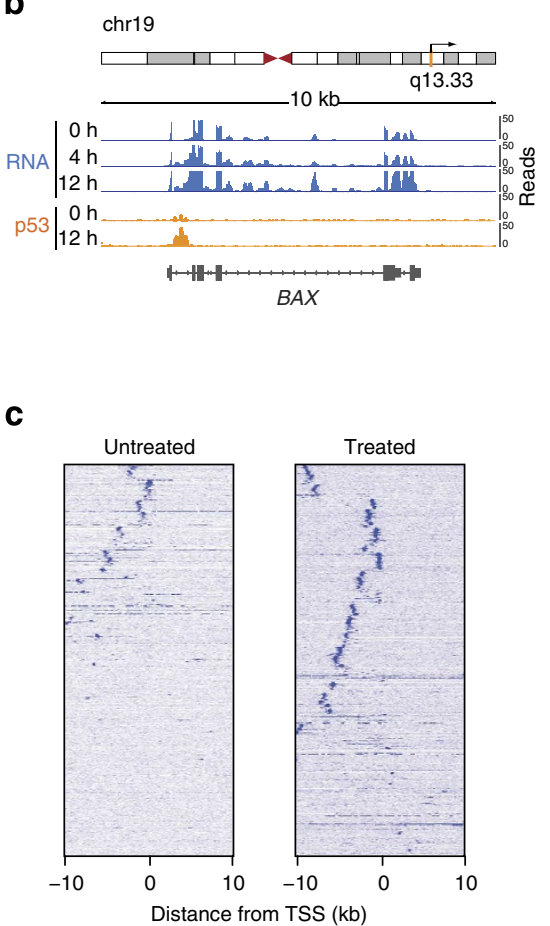

d

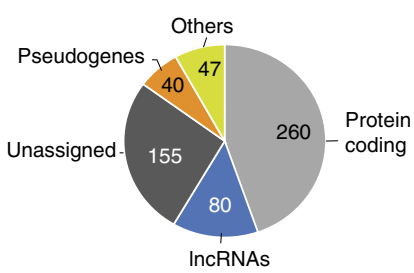

e

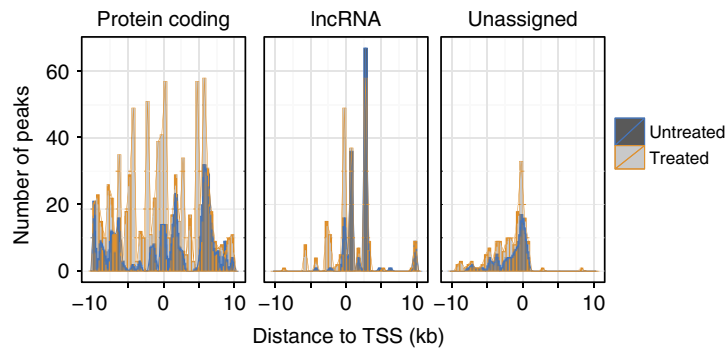

f

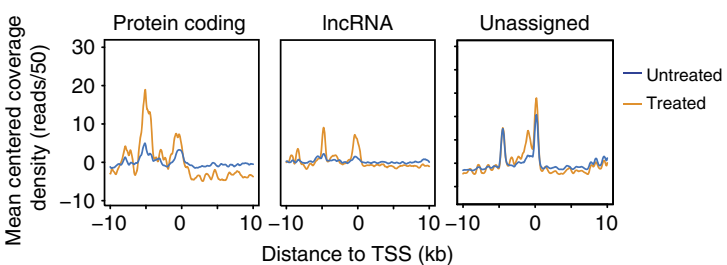

g

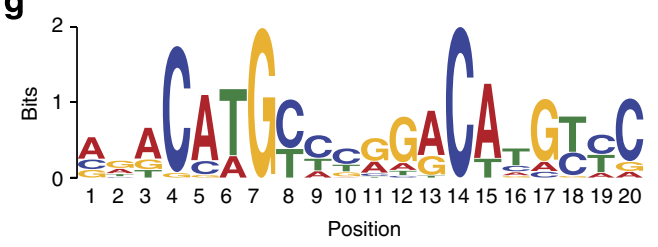

Figure 2 | Genome-wide analysis of p53 binding. (a) Total number of p53-binding loci identified by p53 ChIP-seq analysis of HCT116 cells untreated or treated with DNA damage for $12 \mathrm{~h}$. (b) Schematic representation of the chromosomal location of the BAX gene locus, RNA-seq and p53 ChIP-seq signals at $\mathrm{O}$ and $12 \mathrm{~h}$ of 5 -FU treatment. (c) Density heatmap of the p53-binding sites across $10 \mathrm{~kb}$ from the TSS of genes in untreated and treated HCT116 cells. (d) Distribution by biotypes of genes differentially expressed and bound by p53 upon DNA-damage treatment for $12 \mathrm{~h}$. (e) Number of p53-binding sites and position along $10 \mathrm{~kb}$ from the TSS of the nearest gene separated into protein-coding, IncRNA or unassigned gene loci. (f) Distribution of ChIP-seq reads along $10 \mathrm{~kb}$ of distance from the nearest TSS of the indicated gene types. ( $\mathbf{g}$ ) p53 consensus motif defined by MEME motif analysis of the sequences of p53 ChIP-seq peaks of genes upregulated by DNA damage ( $P=4.9 \mathrm{E}-22$, TOMTOM match statistic).

We focused our attention on the noncoding transcripts differentially expressed on DNA damage and directly regulated by $\mathrm{p} 53$, which we named p53-regulated $\operatorname{lncRNAs}(P R-\ln c R N A s)$. We identified a total of 27 transcripts (from 25 genomic loci; Supplementary Table 4). Eleven of them were annotated by Gencode v19 as intergenic lncRNAs (5), antisense lncRNAs (4) or processed transcripts (2). In addition, we found 16 unassigned transcripts, which in some cases had been annotated in previous studies as lncRNAs (Supplementary Table 4). By analysis of enrichment of chromatin marks, relative position to proteincoding genes and quantification of their coding potential (CPAT $\left.\operatorname{scor}^{28}\right)$, we concluded that, while 7 of the 16 unassigned transcripts could be an artefact of the RNA-seq assembly (for example, extended $3^{\prime}$ untranslated region of the nearest gene), the remaining 9 likely represent p53-regulated lncRNAs.

These results were independently validated by qRT-PCR, observing that 6 out of the 9 unassigned transcripts and the 11 annotated lncRNAs presented the expected expression pattern in p53 WT cells. Furthermore, by analysing their expression in the isogenic HT116 $\mathrm{p}^{-1-}$ cell line ${ }^{22}$, we confirmed that their differential expression is dependent on the presence of $\mathrm{p} 53$ in the cells (Supplementary Fig. 2). A summary table of the p53-regulated lncRNAs (excluding the processed transcripts) and some of their features is presented in Fig. 3a.

To further characterize the p53-regulated lncRNAs, and given the prominent use of mouse models for p53 studies, we wanted to determine the extent of conservation of $\mathrm{p} 53$ regulation in mouse cells. We therefore compared our human p53 ChIP-seq data with previously published p53 ChIP-seq data from mouse embryonic fibroblasts (MEFs) treated with the DNA-damage drug doxorubicin $^{29}$. The genome-wide analysis revealed that $25 \%$ of the p53-binding loci found in the HCT116 human cancer cells were also recognized by p53 in MEFs (Supplementary Table 5). Interestingly, when we quantified only the peaks associated with the differentially expressed genes, the proportion increased to $50 \%$, suggesting that p53 activity on DNA-damage treatment is highly (but not totally) conserved even between different cell types of distant mammalian species.

Interestingly, the transcripts with conserved regulation between mouse and human cells included three noncoding 
a

\begin{tabular}{|c|c|c|c|c|c|c|c|c|c|c|c|}
\hline \multicolumn{2}{|c|}{ Name } & Classification & \multicolumn{3}{|c|}{ RNA expression } & \multicolumn{4}{|c|}{ p53 binding } & \multicolumn{2}{|c|}{ Subcellular localization } \\
\hline \multirow[t]{3}{*}{ Annotation } & Here & & $0 \mathrm{~h}$ & $4 \mathrm{~h}$ & $12 \mathrm{~h}$ & $\mathrm{Oh}$ & $12 \mathrm{~h}$ & MEFs & p53 motif & Nucleus & Cytoplasm \\
\hline & PR-InCRNA-11 & Intergenic & & & & & & & CTACATGTATGGACACGTTG & & \\
\hline & PR-InCRNA-13 & Intergenic & & & & & & & $\begin{array}{ll}\mathrm{N} / \mathrm{A} \\
\end{array}$ & & \\
\hline \multirow[t]{2}{*}{ RP11-206L10.9 } & PR-IncRNA-6 & Intergenic & & & & & & & $\mathrm{N} / \mathrm{A}$ & & \\
\hline & PR-InCRNA-10 & Antisense & & & & & & & CGGCATGCCCGGGCACGCTG & & \\
\hline \multirow{3}{*}{$\begin{array}{l}\text { RP13-631K18.3 } \\
\text { LINC00854 }\end{array}$} & $P R-I n C R N A-3$ & Intergenic & & & & & & & GGGCAAGCTTGGACATGCAC & & \\
\hline & $P R-I n C R N A-8$ & Antisense & & & & & & & $\mathrm{N} / \mathrm{A}$ & & \\
\hline & PR-InCRNA-12 & Intergenic & & & & & & & $\mathrm{N} / \mathrm{A}$ & & \\
\hline \multirow[t]{2}{*}{ RP11-467J12.4 } & $P R-I n C R N A-1$ & Antisense & & & & & & & AAGCATGCCTGGGCAAAGTC & & \\
\hline & PR-IncRNA-17 & Intergenic & & & & & & & N/A & & \\
\hline \multirow{4}{*}{$\begin{array}{c}\text { AC003088.1 } \\
\text { ac-BPGBPG55C20 } \\
\text { RP11-7F17.7 }\end{array}$} & PR-IncRNA-5 & Intergenic & & & & & & & GCACAAGCCCGGGCTTGTCA & & \\
\hline & $P R-\operatorname{InCRNA-4}$ & Intergenic & & & & & & & $N / A$ & & \\
\hline & $P R-I n C R N A-2$ & Intergenic & & & & & & & $\mathrm{N} / \mathrm{A}$ & & \\
\hline & PR-InCRNA-18 & Intergenic & & & & & & & N/A & & \\
\hline RP11-6013.5 & $P R-I n C R N A-7$ & Antisense & & & & & & & $\mathrm{N} / \mathrm{A}$ & & \\
\hline
\end{tabular}

b

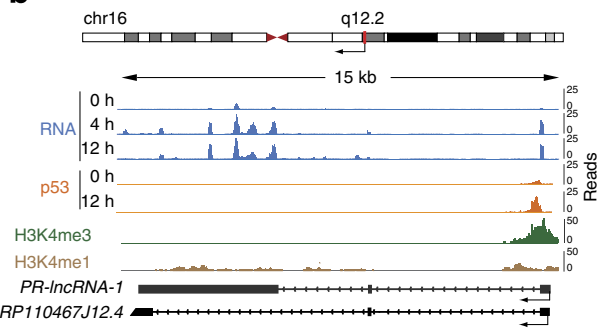

C

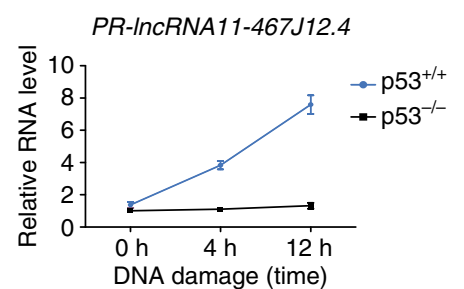

$\mathbf{f}$

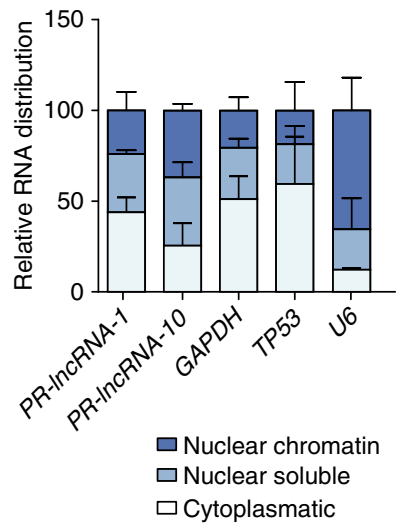

d

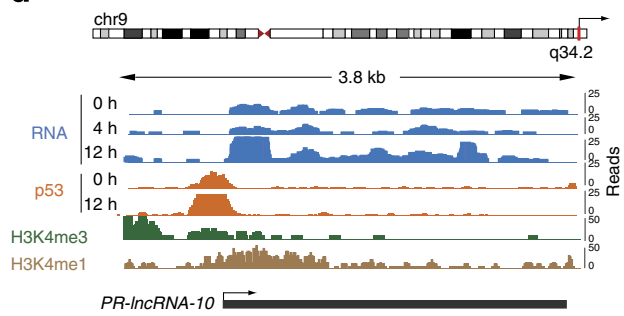

e

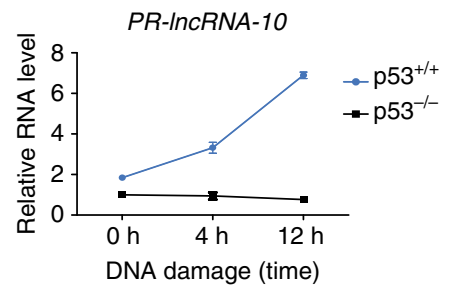

g
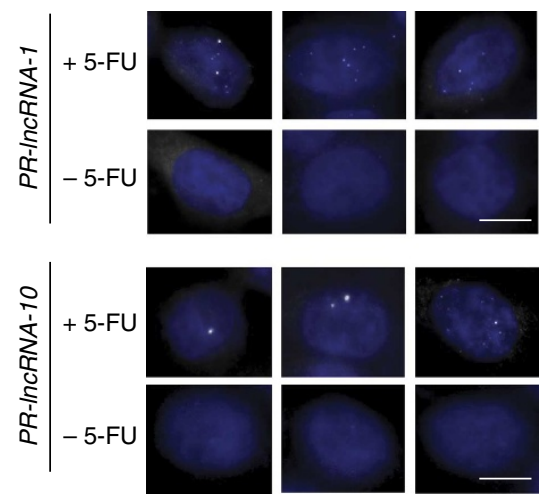

Figure 3 | Human IncRNAs directly regulated by p53. (a) Summary table of IncRNAs directly regulated by p53. Different shades of blue indicate the RNA expression values for each IncRNA under the different conditions of treatment. The presence of p53 binding to the IncRNA loci in HCT116 cells at 0 and $12 \mathrm{~h}$ of 5 -FU treatment or in MEFs treated with doxorubicin is indicated in orange, and the p53-binding sequence motif obtained by MEME analysis is shown. The relative subcellular localization of the IncRNAs determined by subcellular fractionation followed by qRT-PCR is indicated with colours from white to dark blue according to increasing RNA levels. Values are the average of three biological replicates. (b-d) Schematic representation of the chromosomal locations of the PR-IncRNA-1 and PR-IncRNA-10 gene loci. RNA expression (RNA-seq signal), p53, H3K4me3 and H3K4me1 ChIP-seq peaks and transcript structures as assembled by Cufflinks (grey) or annotated by Gencode (black). Validation by qRT-PCR of the PR-IncRNA-1 (c) and PR-IncRNA10 (e) in an independent experiment with HCT116 p53 $3^{/+}$and p53-/- untreated or treated with DNA damage for 4 and $12 \mathrm{~h}$. Values represent the mean \pm s.d. of three biological replicates. (f) Subcellular localization. Percentage of total RNA found in the nuclear fraction bound to chromatin, nuclear soluble and cytoplasmic fractions in the HCT116 p53+/+ cells determined by qRT-PCR. Values represent the mean \pm s.d. of three biological replicates. (g) RNA fluorescence in situ hybridization of PR-InCRNA-1 and PR-IncRNA-10 in HCT116 p53+/+ cells untreated ( -5 -FU) or treated (+5-FU) with 5-FU for $12 \mathrm{~h}$. White line indicates $10 \mu \mathrm{m}$.

RNAs (two lncRNAs and one processed transcript; Supplementary Table 5; Fig. 3a). To confirm this observation, we performed $\mathrm{qRT}-\mathrm{PCR}$ in $\mathrm{p} 53^{+/+}$and $\mathrm{p} 53^{-/-}$MEFs treated with doxorubicin on the mouse orthologous regions corresponding to the noncoding RNAs. For all of them, we confirmed higher RNA expression in the presence of p53 and increased expression upon DNA-damage treatment (Supplementary Fig. 3g). 
In summary, we have identified a high-confidence set of 18 lncRNAs that are directly regulated by p53 upon DNA-damage treatment in human cells, 3 of which show conserved regulation in mouse cells.

p53-regulated lncRNAs contribute to the p53 transcriptional network. After identifying human IncRNAs that are bona fide p53 transcriptional targets, we wanted to determine to what extent they contribute to the gene expression changes caused by p53 upon DNA damage. We selected two representative lncRNAs: (i) $P R-\ln c R N A-1$, a 5,250-nt-long multiexonic intergenic transcript, annotated by Gencode as RP11-467J12.4, predominantly localized in the nucleus of cells and shown to be p53 regulated in both human and mouse cells (Fig. 3a-c,f,g) and (ii) $P R-\ln c R N A-10$, a non-annotated monoexonic intergenic lncRNA of 2,780 nt of length, localized in the nucleus and with no detected p53 regulation in mouse (Fig. $3 \mathrm{a}, \mathrm{d}-\mathrm{g}$ ).

To deplete cells of these lncRNAs, we designed several antisense oligonucleotides (ASOs) to achieve specific degradation and selected the two most effective ASOs in reducing the lncRNA levels (Fig. 4a,b). For each lncRNA, we then transfected HCT116 cells with two targeting ASOs, as well as a non-targeting ASO as control for $36 \mathrm{~h}$ and then treated the cells with 5 -FU for $12 \mathrm{~h}$ to induce p53. Total RNA was isolated and gene expression analysis was performed by microarray (See Methods and Suplementary Methods).

The analysis revealed that the depletion of $P R-\ln c R N A-1$ affected the expression of 932 genes (748 up- and 184 downregulated), while $P R-\ln c R N A-10$ reduction caused the altered expression of 1,647 genes (1,290 up- and 357 downregulated; $B>0$, limma B-statistics or log odds; Supplementary Table 6). Interestingly, the relationship between p53 and these two lncRNAs was confirmed by the prediction of p53 as a significant upstream regulator for both sets of genes $(P=2.12$ $\mathrm{E}-05$ for $P R-\ln c R N A-1$ and $P=1.00 \mathrm{E}-12$ for $P R-\ln c R N A-10$, Ingenuity Fisher's exact test). The gene expression changes observed by microarray analysis were validated in independent experiments where we transfected separately the individual ASOs to knockdown the lncRNAs and performed qRT-PCR for a panel of representative genes. We confirmed the expression changes for 12 out of 17 (PR-lncRNA-1) and 16 out of 18 (PR-lncRNA-10) differentially expressed genes. Few mRNAs that did not show a statistically significant differential expression by qRT-PCR, still showed the same trend (up- or downregulated) observed in the microarray analysis (Supplementary Figs $4 \mathrm{a}$ and 5a). In addition, we performed independent validations by transfecting the ASOs into HCT116 $\mathrm{p} 53^{-/-}$cells with or without DNA-damage treatment. Under these conditions, we could not detect any effect of the ASO treatment on the validation gene set, confirming that the effects observed are specific of the p53-dependent expression of $P R-\ln c R N A-1$ and PR-lncRNA-10 (Supplementary Figs $4 \mathrm{~b}, \mathrm{c}$ and $5 \mathrm{~b}, \mathrm{c})$.

We next compared the effect of PR-lncRNA-1 and PR-lncRNA10 depletion with the observed gene expression changes caused by DNA damage in HCT116 cells. Out of the 69 protein-coding genes regulated by $P R-\ln c R N A-1$ that consistently changed with DNA-damage treatment, many were related to induction of apoptosis and proliferation. For instance, we found the apoptosis regulators $B C L 2 L$ and $B I R C 3$, the DNA polymerase subunit POLA1 or the growth factor TGFB2 (Fig. 4c,d; Supplementary Table 7). On the other hand, 109 of the protein-coding genes regulated by $P R-\operatorname{lnc} R N A-10$ constituted another component of the DNA-damage response, and included the cell cycle inhibitor CDKN1A, the transcription factor JUNB or the apoptosis regulators BIRC6, TP53I3 and FAS among many others
(Fig. 4c,d; Supplementary Table 7). In agreement with a potential role of these lncRNAs in the p53 response to DNA damage, p53 was found to be the most significant predicted upstream regulator and enriched pathway for the sets of genes affected by both DNA damage and $P R-\ln C R N A-1$ or $P R-\ln c R N A-10(P=1.0 \mathrm{E}-07$ or $P=2.1 \mathrm{E}-03$, respectively). This is illustrated by the network depicted in Fig. 4d, which represents the predicted relationships between some of the genes co-regulated by p53 and PR-lncRNA-1 or PR-lncRNA-10.

Altogether, the results shown here suggest that $P R-\ln c R N A-1$ and $P R-\ln c R N A-10$ are active components of the p53 transcriptional response and that they modulate the gene expression response to DNA damage downstream of p53.

$P R$ - $\ln c R N A s$ required for efficient binding of p53 to gene targets. Our results showed that PR-lncRNA-1 and PR-lncRNA-10 are not just directly regulated by $\mathrm{p} 53$, but are involved in the regulation of genes of the p53 pathway. We then investigated how these lncRNAs could affect the expression of their targets. While the microarray analysis showed changes in the steady-state levels of the mRNAs regulated by $P R-\ln c R N A-1$ and $P R-\ln c R N A-10$, it did not distinguish whether the changes are taking place at the transcriptional or post-transcriptional level. To address this point, we analysed the stability of $P R-\operatorname{lncRNA-1}$ and $P R-\ln c R N A-10$ target mRNAs upon knockdown of the lncRNAs by blocking their transcription with actinomycin-D treatment. These experiments did not show significant changes in the stability of the mRNAs analysed, suggesting that $P R-\ln c R N A-1$ and $P R-\ln c R N A-$ 10 do not act post-transcriptionally on these mRNAs, but rather regulate their expression at the transcriptional level (Supplementary Fig. 6).

As discussed above, the transcription factor p53 is predicted to be the upstream regulator of the genes affected by $P R-\ln c R N A-1$ and $P R-\ln c R N A-10$ knockdowns. We therefore hypothesized that $P R-\ln c R N A-1$ and $P R-\ln C R N A-10$ could affect p53 activity. We excluded the possibility that p53 gene expression was affected, as the microarray analyses and additional qRT-PCR validations clearly showed that p53 mRNA levels were not changed on $P R$ lncRNA-1 nor PR-lncRNA-10 knockdown (Supplementary Figs 4 and 5). As p53 is tightly regulated at the protein level, we analysed total p53 protein level in $P R-\ln c R N A-1$ and $P R-\ln c R N A-10$ knockdown conditions. However, we did not detect any differences upon knockdown of the lncRNAs (Fig. 5a,b). Similarly, we did not detect any changes in the levels of phosphorylated $\mathrm{p} 53$ protein at serine 15 , which is generally thought to be involved in the activation of p53 after DNA damage $^{30}$. We concluded that neither PR-lncRNA-1 nor PR-lncRNA-10 affects p53 protein levels or phosphorylation.

We then tested whether the ability of p53 to transcriptionally activate some target genes could be influenced by $P R-\ln c R N A-1$ or $P R-\operatorname{lncRNA-10}$. To that end, we selected a set of genes that are p53 direct transcriptional targets, that is, are directly bound by p53 and change their expression on DNA damage (SERPINB5, FAS, CDKN1A, BCL2L1, BBC3, BAX and MDM2; see Supplementary Table 3 ). Most of the selected genes (SERPINB5, $F A S, C D K N 1 A$ and BCL2L1) were also found altered in our microarray analysis by the knockdown of $P R-\ln c R N A-1$ and/or PR-lncRNA-10 (Supplementary Table 8; Fig. 4c,d). We then performed p53 ChIP in the presence and absence of $P R-\ln c R N A-1$ or $P R-\ln C R N A-10$ depletion. On $P R-\ln c R N A-1$ and/or $P R$ IncRNA-10 depletion, we observed a significant decrease in the binding of p53 to the p53REs of SERPINB5, CDKN1A, BCL2L1 and $B B C 3$ genes, although the decrease was generally more pronounced on $P R-\ln c R N A-10$ inhibition (Fig. 5c). Furthermore, when the mRNA levels were quantified in the same experiments, 
a

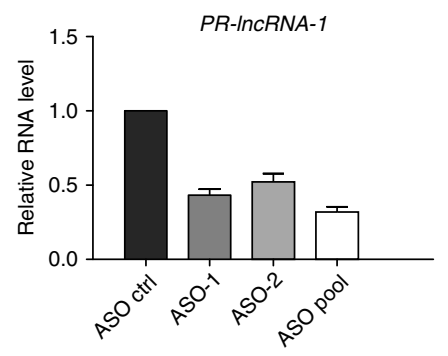

b

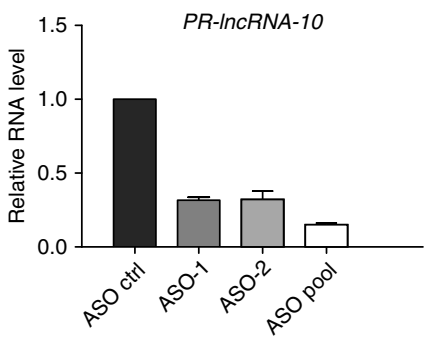

C

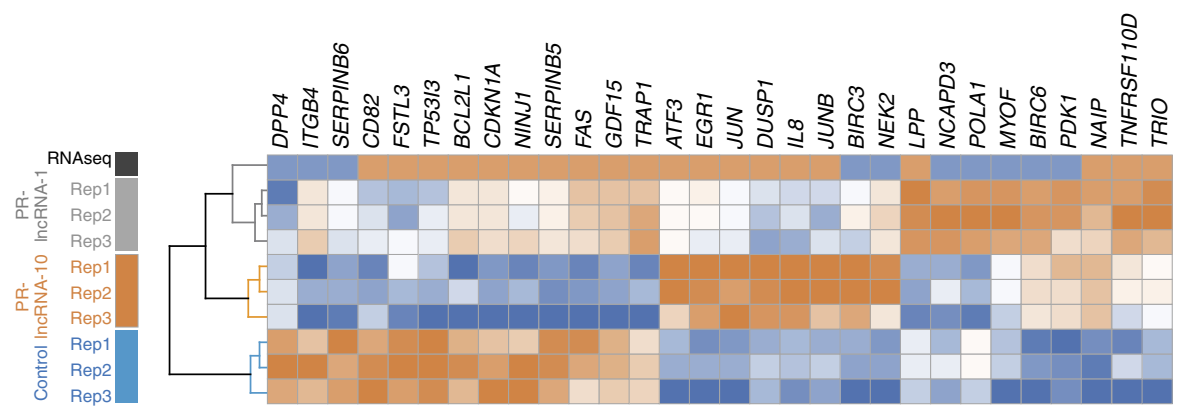

d
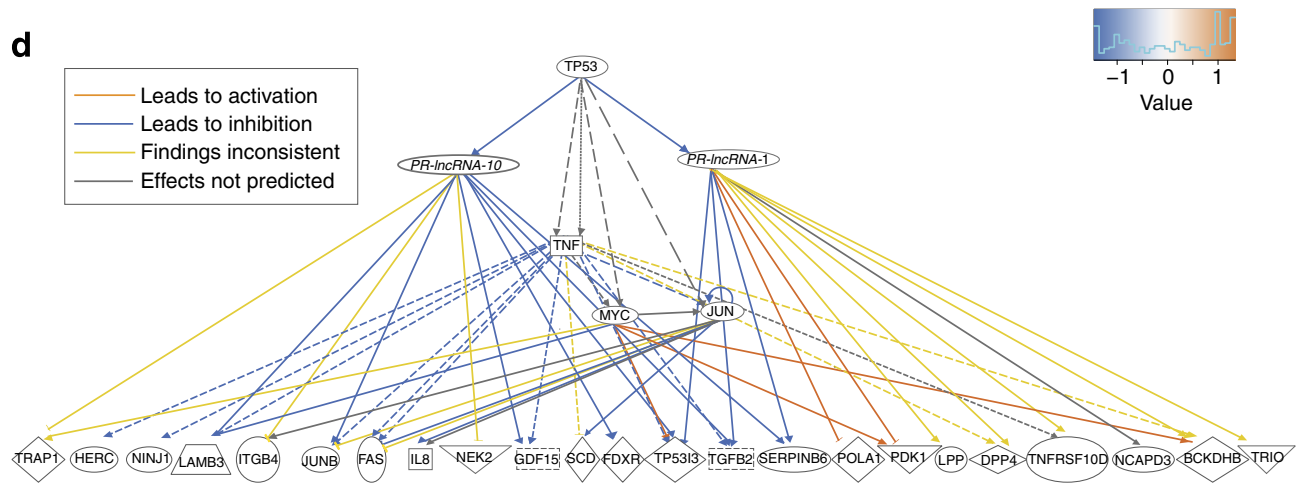

Figure 4 | Role of the p53 targets PR-IncRNA-1 and PR-IncRNA-10 in the p53 transcriptional response. (a,b) HCT116 cells were transfected with a non-targeting ASO (ASO Ctrl) or with two different ASOs (ASO-1 and ASO-2) targeting PR-IncRNA-1 (a) or PR-IncRNA-10 (b), separately or in combination (ASO pool). PR-IncRNA-1 and PR-IncRNA-10 RNA knockdown efficiencies were determined by qRT-PCR. Graphs represent the mean ( \pm s.d.) of three independent experiments. (c) Expression levels of representative genes found differentially expressed in at least one of the microarray analysis (PR-IncRNA1 or PR-IncRNA-10 depletion) and in HCT116 when treated with 5-FU (RNA-seq analysis). Colours from dark blue to orange indicate increasing RNA levels in each of the experiments. (d) Network connecting p53, PR-IncRNA-1 and PR-IncRNA-10 with some of the commonly regulated genes involved in the DNA-damage response as predicted by Ingenuity Pathway Analysis.

we observed a decrease in the expression of the genes in correlation with the decrease in $\mathrm{p} 53$ binding to their promoters upon the lncRNAs depletion (Fig. 5d).

Taken together, these results suggest that $\mathrm{p} 53$ requires $P R-\ln c R N A-10$ and, in lesser extent, PR-lncRNA-1 to efficiently bind and activate some of its direct transcriptional targets.

$P R-\ln c R N A-1$ and $P R-\ln c R N A-10$ are negative regulators of cell survival and proliferation. The gene expression analysis revealed that $P R-\ln c R N A-1$ and $P R-\ln c R N A-10$ modulate the expression of several genes related to cell cycle control and apoptosis induction, which are the major functional outcomes of p53 activation. To determine the role of the p53-regulated lncRNAs in this context, we monitored cell proliferation after depletion of $P R-\ln c R N A-1$ or $P R$-lncRNA-10 in the presence or absence of DNA-damage induction. HCT116 cells were treated with doxorubicin (doxo) to induce DNA damage, as this drug induces expression of p53 and its target genes, including $P R-\ln c R N A-1$ and $P R-\ln C R N A-10$ (Supplementary Fig. 7a,b), but is not as strong apoptosis inducer as 5-FU, allowing us to carry out experiments for several days. Cell proliferation assays were performed with HCT116 cells showing a significant increase in the number of viable cells when depleted of $P R-\ln c R N A-1$ or $P R-\ln c R N A-10$ compared with the controls (Supplementary Fig. 7c,d), both in the presence and absence of drug treatment, although the difference in proliferation between lncRNA-depleted cells and controls was more marked in cells treated with DNA damage (Fig. 6a,b). These results suggested that both $P R-\ln c R N A-1$ and $P R-\ln c R N A-10$ may contribute to the p53 pro-apoptotic and/or cell cycle regulatory functions.

The major effect of DNA damage on HCT116 cells is a generalized cellular apoptosis, which reaches $\sim 40 \%$ within $12 \mathrm{~h}$ of 5-FU treatment (Fig. 6, Supplementary Fig. 5e). To evaluate the role of the lncRNAs under investigation in this cellular mechanism, we quantified the number of apoptotic cells following depletion of $P R-\ln c R N A-1$ or $P R-\ln C R N A-10$ under DNA-damage conditions. Consistent with the effect observed in proliferation, we found a significant decrease in the number of apoptotic cells measured by annexin $\mathrm{V}$ detection, reaching close to $50 \%$ re- 
a

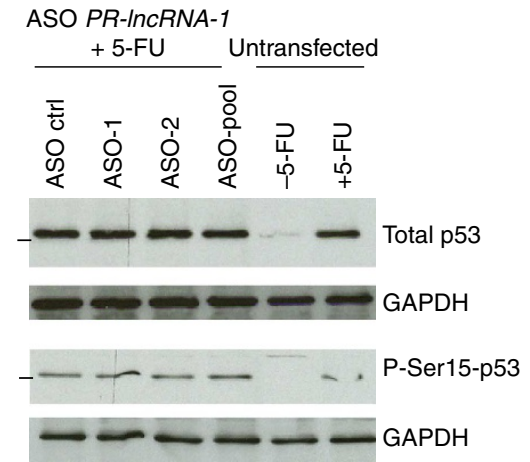

b

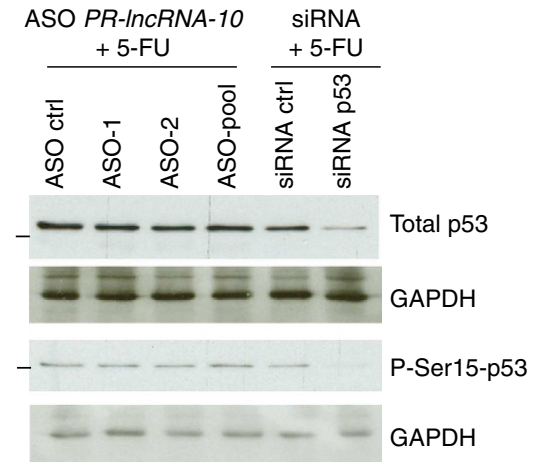

C
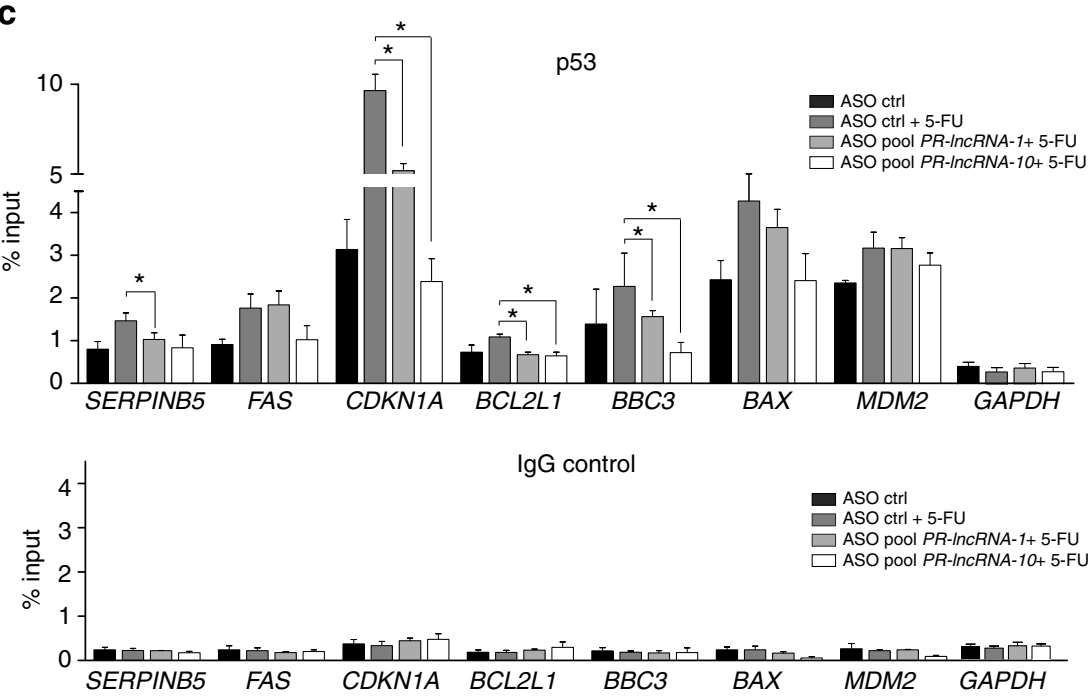

d

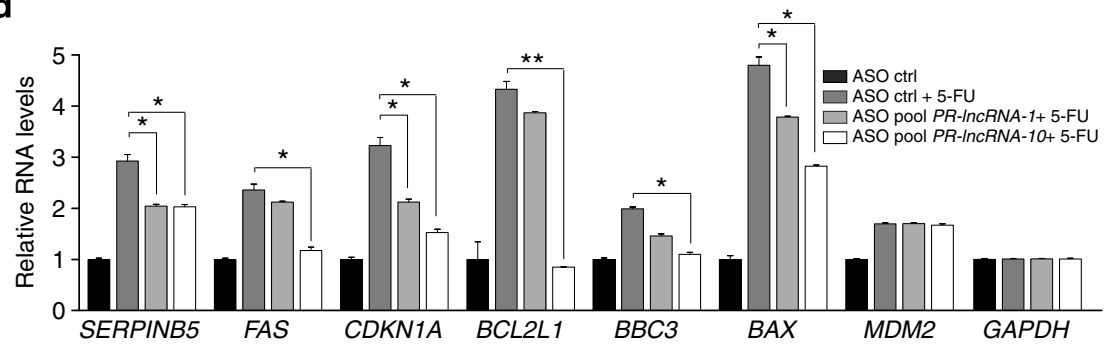

Figure 5 | PR-IncRNA-1 and PR-IncRNA-10 are required for the transcriptional activation of some genes by p53. (a,b) Western blot analysis of total p53 and phospho-p53 (Serine 15) on HCT116 cells transfected with ASOs for PR-IncRNA-1 (a) or PR-IncRNA-10 depletion (b) and treated as indicated. GAPDH is shown as a loading control. MW mark indicates $50 \mathrm{kDa}$. (c) p53 ChIP of p53 direct target genes regulated by PR-IncRNA-1 and PR-IncRNA-10. ChIP enrichment of $\mathrm{p} 53$ and control IgG of the indicated loci in HCT116 cells after the transfection with ASO pool for PR-IncRNA-1, PR-IncRNA-10 or ASO control for $36 \mathrm{~h}$ and treatment with 5-FU for $12 \mathrm{~h}$. GAPDH promoter was included as a negative control. The mean \pm s.d. of three biological replicates, and the significant differences relative to the condition ASO ctrl +5-FU are shown. (d) Relative expression levels of the p53 direct target genes regulated by PR-InCRNA-1 and PR-InCRNA-10 in HCT116 cells treated like in c and determined by qRT-PCR. Values are the average of three biological replicates, and the significant differences relative to the condition ASO ctrl $+5-\mathrm{FU}$ are shown. All graphs $(\mathbf{c}, \mathbf{d})$ represent the mean $( \pm \mathrm{s}$.d.) of at least three independent experiments. Significance was determined by two-tailed unpaired $t$-test. ${ }^{\star} P<0.05$ and ${ }^{\star \star} P<0.01$.

duction under the best knockdown conditions (Figs $6 \mathrm{c}$ and $4 \mathrm{a}$; Supplementary Fig. 7f,g). This effect was more pronounced when PR-lncRNA-1 was inhibited compared with PR-lncRNA-10 inhibition (55 and $43 \%$ of reduction, respectively), and was confirmed by quantifying caspase $3 / 7$ levels under similar experimental conditions (Fig. 6d,e). We therefore concluded that $P R$-lncRNA-1 and PR-lncRNA-10 contribute to apoptosis induction by DNA damage.

p53 activity as a tumour suppressor involves also a tight control of cell cycle progression, p53 being able to control both G1 and G2/M checkpoints ${ }^{31}$. To further characterize the biological role of the two p53-regulated lncRNAs, we carried out cell cycle analysis of HCT116 cells depleted of the lncRNAs both in the presence or absence of DNA damage (doxo or 5-FU). When either PR-lncRNA-1 or PR-lncRNA-10 was depleted, and in all the experimental conditions tested, we observed a significant increase of cells in S-phase of cell cycle consistent with the increase in cell proliferation observed under the same conditions (Fig. 6f-i; Supplementary Fig. 7h,i). As for the cell proliferation assays, the differences observed in the cell cycle phase distribution 
a

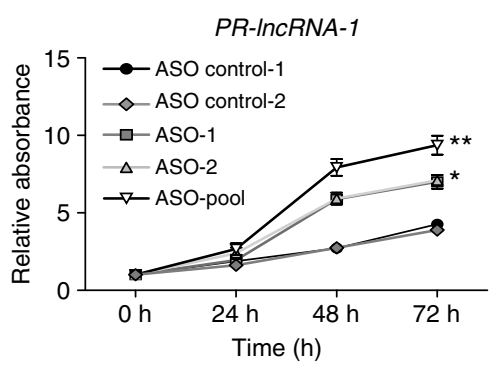

c
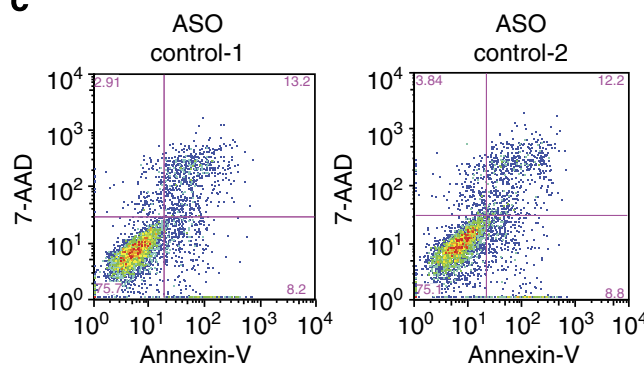

d

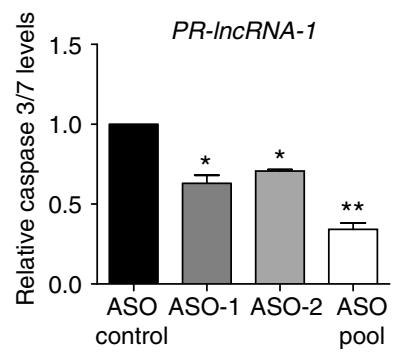

f

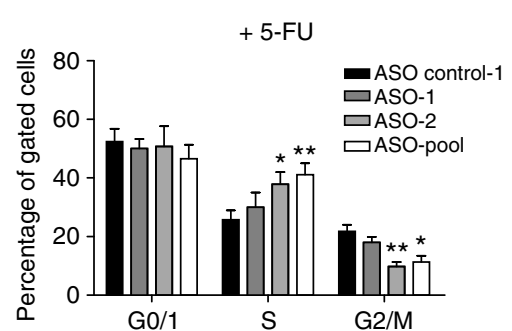

h

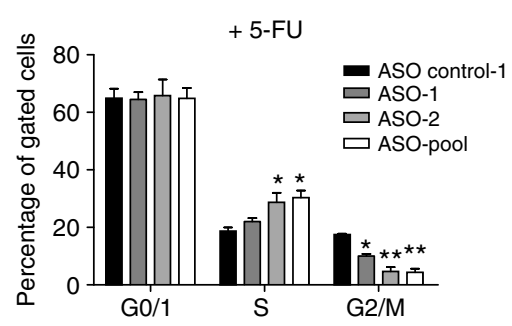

b
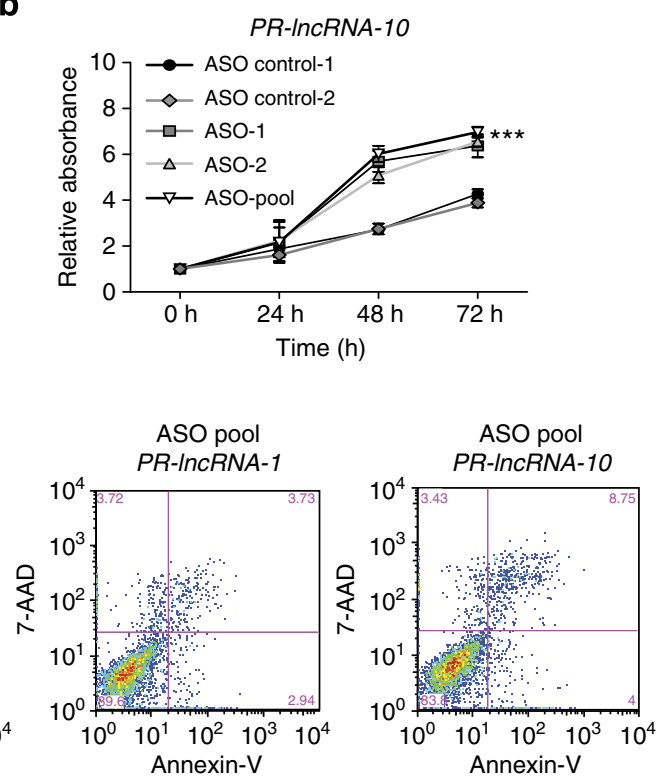

e

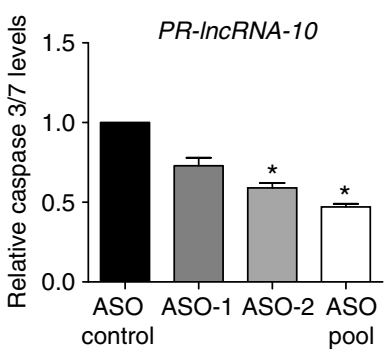

g

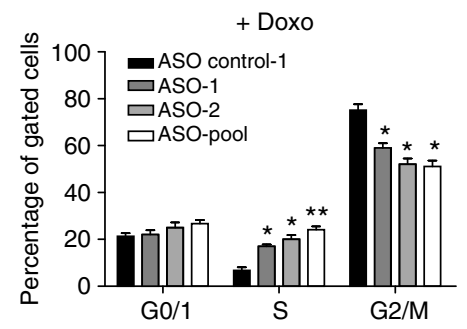

i

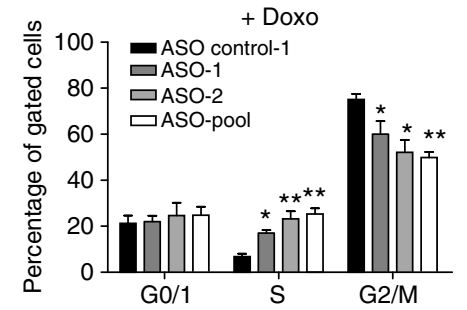

Figure 6 | PR-IncRNA-1 and PR-IncRNA-10 modulate cell proliferation and apoptosis. (a,b) HCT116 cells were transfected with two separate non-targeting ASOs (Ctrl-1 and Ctrl-2) or with two different ASOs (ASO-1 and ASO-2) targeting PR-IncRNA-1 or PR-IncRNA-10, separately or in combination (ASO pool), and treated with $150 \mathrm{nM}$ DNA-damage Doxo for $12 \mathrm{~h}$. Cell proliferation was measured by MTS assay up to $72 \mathrm{~h}(\mathbf{a}, \mathbf{b})$. (c) Percentage of apoptotic cells measured by annexinV-7AAD (7-aminoactinomycin D) staining on cells transfected with the indicated ASOs and treated with the $350 \mu \mathrm{M}$ DNA-damage drug 5-FU for $12 \mathrm{~h}$. (d,e). Apoptosis was determined by quantification of caspase $3 / 7$ levels in cells treated with 5 -FU for $12 \mathrm{~h}$ and transfected with the indicated ASOs. (f-i) Cell cycle phase distribution analysed by propidium iodide staining of cells transfected with ASOs specific for $P R-I n C R N A-1(\mathbf{f}, \mathbf{g})$ or PR-IncRNA-10 (h,i) depletion and treated with the indicated drugs for $12 \mathrm{~h}$. All graphs $(\mathbf{a}-\mathbf{i})$ represent the mean ( \pm s.d.) of at least three independent experiments. Significance was determined by two-tailed unpaired $t$-test. ${ }^{\star} P<0.05$ and ${ }^{\star \star} P<0.01$. 
between lncRNAs-depleted cells and controls were more pronounced following DNA damage compared with untreated cells (Fig. 6f-i; Supplementary Fig. 7h,i). These results suggest that both $P R-\ln c R N A-1$ and $P R-\ln c R N A-10$ contribute to cell cycle regulation, playing a role even when expressed at basal levels, as observed with cells not treated with DNA-damage drugs. However, their roles in cell cycle progression appeared more pronounced under DNA-damage conditions, suggesting a major role for the lncRNAs in the DNA-damage response.

Altogether, we show that the p53-regulated PR-lncRNA-1 and $P R-\ln c R N A-10$ contribute to the biological outcome of the p53 pathway activation by promoting apoptosis and cell cycle arrest.

PR-lncRNAs constitute a tumour suppressor signature. p53 malfunction is well known to play a major role in the development of cancer. A total of $60 \%$ of non-hypermutated tumours harbour mutations in the p53 gene $\mathrm{g}^{32,33}$, and $70 \%$ of colorectal carcinomas show loss of heterozygosity in $17 \mathrm{p}$, where p53 gene locus resides ${ }^{34,35}$.

The results obtained for $P R-\ln C R N A-1$ and $P R-\ln c R N A-10$ suggest their involvement in the p53 tumour suppression function in colorectal cancer cells. We therefore hypothesized that their expression levels, as well as the expression of other p53regulated lncRNAs, could be altered in human primary colorectal cancer specimens. To corroborate this hypothesis, we analysed the expression of the p53 directly regulated lncRNAs identified in this study in a cohort of human colon adenocarcinoma healthy tissue-paired samples.

In agreement with a potential role of the lncRNAs in tumour suppression, the expression levels of all the lncRNAs analysed were lower in colorectal tumours compared with normal tissue, although changes only reached significance $(P<0.01)$ for 7 out of 12 lncRNAs (Fig. 7a).

To confirm that the low expression levels of the p53-regulated lncRNAs are a distinct feature of the tumour cells, we quantified the sensitivity and specificity of the lncRNAs as biomarkers by performing receiver operator characteristic (ROC) analysis (see Methods). The expression levels of five p53-regulated lncRNAs (PR-lncRNA-1, PR-lncRNA-2, PR-lncRNA-10, PR-lncRNA-17 and $P R-\ln c R N A-18)$ showed a statistically significant classificatory value (area under the curve $(\mathrm{AUC})<0.7$ ) that distinguished tumour from normal tissue (Fig. $7 \mathrm{~b}$ ).

Next, to improve the classification performance of the 5-lncRNA-signature, we applied a machine-learning algorithm based on logistic regression (see Methods). The higher classificatory power of the lncRNA signature was confirmed by an AUC value of 0.921 for the tenfold analysis, with a $P$ value $=1.9 \mathrm{e}-12$ for a logistic regression model (Fig. $7 \mathrm{~b}, \mathrm{c}$ ). These results suggest that the expression levels of this p53-regulated lncRNAs could have a diagnostic value in colorectal cancer patients.

\section{Discussion}

Since the realization of p53's pivotal role as a tumour suppressor, a large body of literature has identified p53-regulated genes. However, the p53 network has mostly been assembled one gene at a time and mainly focusing on genes that encode for proteins.

As a closer attempt to assess the activity of p53 on noncoding regions, a recent study has utilized p53 ectopic overexpression to relate the activity of p53 family members to the regulation of a limited number of $\operatorname{lncRNAs}{ }^{36}$. However, genome-wide studies linking the activity of endogenous p53 to the expression of lncRNAs from annotated as well as non-annotated regions of the genome are still missing. Our study provides, for the first time to our knowledge, a global overview of the relationship between p53 activity and expression of lncRNAs in a human cancer cell line in response to DNA-damage treatment. We show that DNA damage inflicted by 5 -FU affects the expression of hundreds of lncRNAs, a proportion similar to that found for protein-coding genes, including a large number of transcripts not previously annotated by Encode. While many of these unassigned transcripts may be artefacts of the RNA-seq assembly, others are shown to be novel lncRNAs expressed under our experimental conditions. Multiple lines of evidence support this idea: (i) some of the unassigned transcripts are annotated in other databases different from Encode $^{5}$, (ii) their TSS is marked by histone modifications associated with active chromatin, (iii) the expression pattern and p53 regulation of several of them has been independently validated and (iv) their expression is suppressed in tumour samples compared with normal tissue.

A small proportion of the lncRNAs altered by DNA damage were found directly bound by $\mathrm{p} 53(\sim 3 \%)$, suggesting that the rest of differentially expressed lncRNAs are likely secondary targets of p53 activation or IncRNAs regulated by DNA damage in a p53independent manner. However, this could be a conservative estimate, as we only considered p53-biding sites that are localized within less than $10 \mathrm{~kb}$ of distance to the TSS, which can be more unequivocally assigned to a gene. This approach defined a high-confidence set of 18 lncRNAs that are bona fide p53 transcriptional targets.

A number of studies have shown that lncRNAs present lower conservation than protein-coding genes across species ${ }^{5,6}$. In our study, we looked for conservation between human and mouse in terms of p53 regulation and found that only 3 of the 18 p53regulated human lncRNAs appeared to be similarly regulated in murine embryonic fibroblasts. Although this low number could be due to the differences between cell types and experimental conditions, it remains possible that some of the human lncRNAs could account for the known divergences between the murine and human $\mathrm{p} 53$ responses ${ }^{37}$. It has been proposed in fact that the more rapidly evolving lncRNA sequences may account for acquired novel biological traits ${ }^{38}$. On the other hand, those lncRNAs that are conserved could represent essential elements of the mammalian p53 response to DNA damage.

As representative of the p53-regulated lncRNAs, we selected PR-lncRNA-1 and PR-lncRNA-10 for further analyses. We found similarities and differences between both $\operatorname{lncRNAs}$. PR- $\ln c R N A-1$ and $P R-\ln C R N A-10$ are mainly localized in the nucleus of the cells. While the regulation of $P R-\ln c R N A-1$ by $\mathrm{p} 53$ appears to be conserved in mouse, $P R-\ln c R N A-10$ is a primate-specific $\ln c \mathrm{RNA}$. Each lncRNA regulates the expression of a different, partially overlapping, set of genes of the p53 network, contributing to the apoptotic response caused by DNA damage. Our data suggest that $P R-\ln c R N A-10$ and, to a lesser extent, $P R-\ln c R N A-1$ modulate the transcriptional activity of $\mathrm{p} 53$ by affecting its ability to recognize some of its binding sites. Although we cannot exclude other possibilities, we speculate that these nuclear PR-lncRNAs may affect the chromatin conformation at these loci, rendering them more accessible to p53 binding. This represents a novel positive-feedback mechanism in which lncRNAs induced by p53 fine-tune p53 transcriptional activity under DNA-damage conditions. However, the precise molecular mechanisms by which PR- $\ln c R N A-1$ and PR- $\ln C R N A-10$ contribute to enhance p53 binding to these gene loci remain to be defined.

In agreement with the results obtained with the HCT116 cell line suggesting a potential role of the lncRNAs as part of the p53 tumour suppressor response, the expression levels of seven p53regulated lncRNAs (including $P R-\ln c R N A-1$ and $P R-\ln c R N A-10$ ) were found significantly decreased in a cohort of colorectal cancer patient samples. This is in part an expected result since p53 gene presents alterations in the vast majority of colorectal tumours ${ }^{39}$. However, this observation confirms our results concerning the role 
a

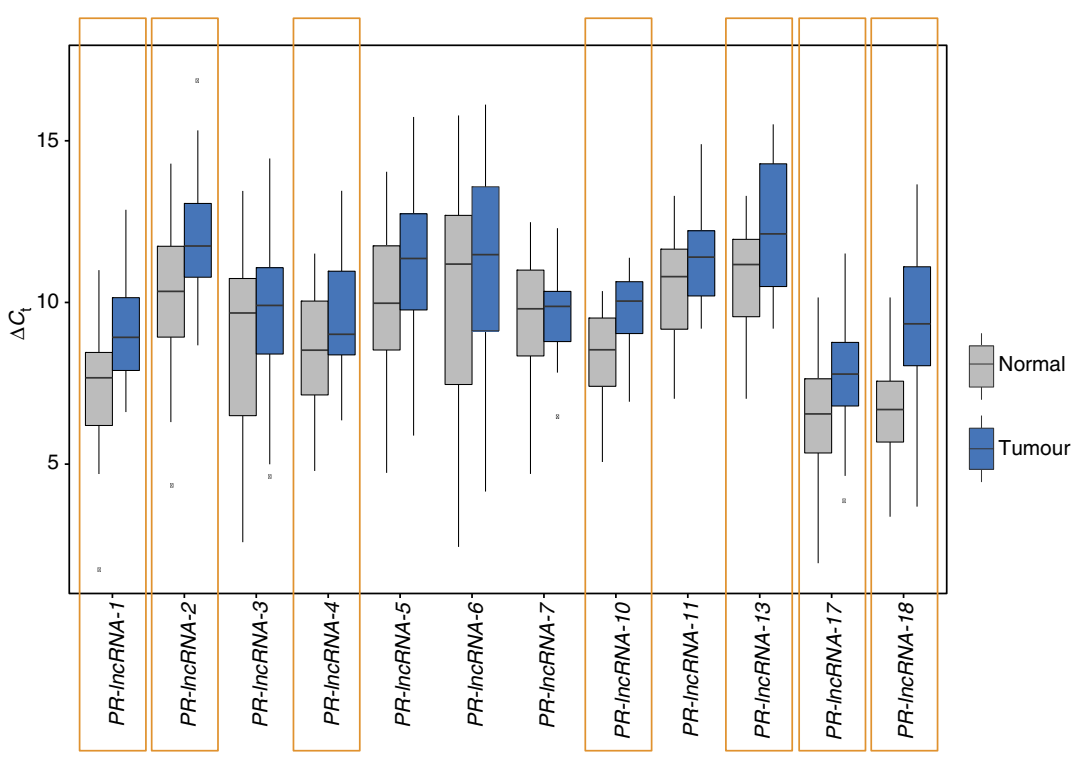

b
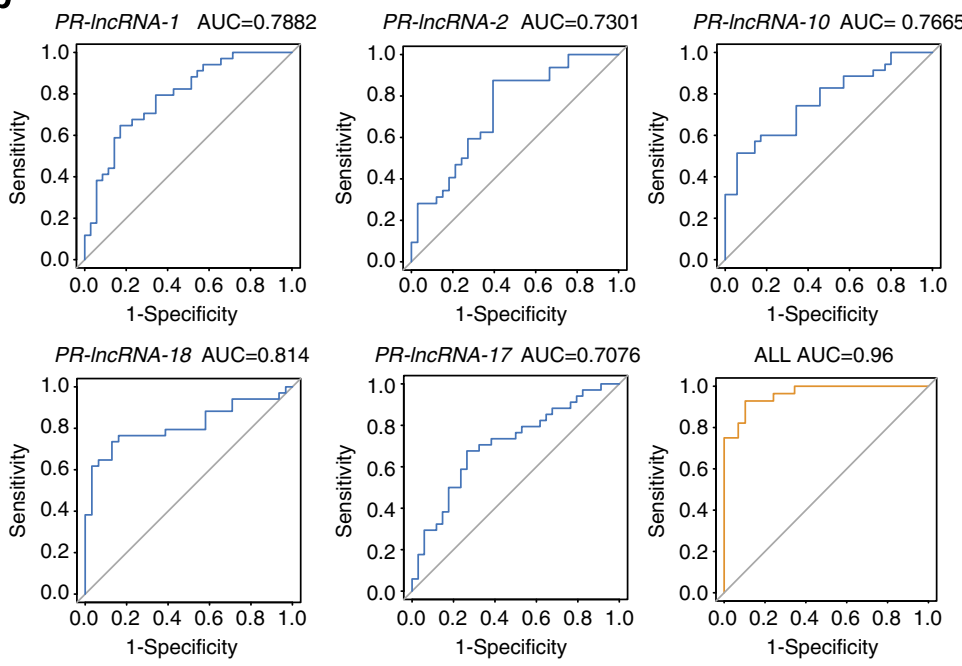

C

- Tumour $\square$ Normal

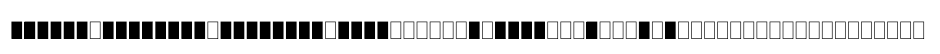

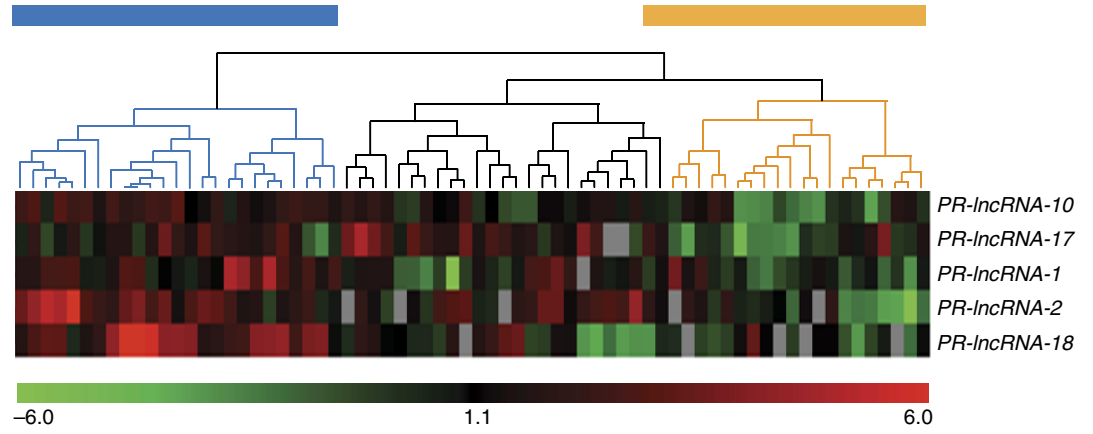

Figure 7 | p53-regulated IncRNAs constitute a tumour-suppressor signature in colorectal cancer. (a) Relative expression level of p53-regulated IncRNAs in a cohort of 35 tumour samples compared with the normal peripheral tissue. The highlighted candidates are significantly downregulated in the tumours $(P<0.01)$. (b) ROC analysis of the sensitivity and specificity for the training cohort for the seven significant p53-regulated IncRNAs. Multivariate ROC analysis for the five p53-regulated IncRNAs with AUC values >0.7. (c) Clustering of the expression levels of the p53-regulated IncRNAs with AUC values $>0.7$. Rows represent IncRNAs and columns represent patients. 
played by the lncRNAs identified in this study in the p53-dependent tumour suppressor response and supports their clinical relevance.

Importantly, the regulation by p53 of these lncRNAs is not exclusive of the human cell line used in this study. We found that, similarly to that observed in HCT116 cells, the expression of the p53-regulated lncRNAs is dependent on p53 and DNA damage in other human cells analysed, including A549 human lung cancer cells (Supplementary Fig. 8a,b) and normal cells (IMR90 and BJ human fibroblasts). This observation, together with the decreased expression in colorectal tumours, leads us to conclude that the lncRNAs identified in this study are involved in the human p53 response independently of the tissue and represent a genuine subset of p53 target genes likely implicated in the tumour formation of all cancer types where p53 is affected.

\section{Methods}

Cell lines and DNA-damage treatment. The colon carcinoma cell lines HCT116 p53 ${ }^{+I+}$ and $\mathrm{p}^{-I-}$ were kindly provided by Dr Vogelstein's laboratory from Johns Hopkins University, Baltimore (USA).

To obtain $\mathrm{p}^{+1+}$ and $\mathrm{p} 53^{-I-}$ MEFs, p53 $3^{\mathrm{LSL} / \mathrm{LSL}}$ MEFs were isolated from embryos of the $\mathrm{p} 53^{\mathrm{LSL} / \mathrm{LSL}}$ mouse strain and infected with AdenoCre or AdenoGFP virus for $12 \mathrm{~h}$ (University of Iowa) at a multiplicity of infection 5 .

For DNA damage, cells were treated with $350 \mu \mathrm{M}$ 5-FU (F6627; Sigma) or $500 \mathrm{nM}$ doxorubicin hydrochloride (D1515; Sigma) for the indicated times.

Western blot. The following antibodies were used for western blot: anti-p53 AB(DO-1) sc-126, dilution 1:1,000; anti-pP53 (S15) Cell Signaling Ref:11/2012, dilution 1:1,000; and anti-GAPDH mAbcam 9484, dilution 1:2,000. Uncropped western blot images are shown in Supplementary Fig. 9.

RNA-seq analysis. The RNA-seq libraries were prepared from purified poly-A ${ }^{+}$ RNA from untreated and 5-FU-treated p53 ${ }^{+/}+$HCT116 cells for 4 and $12 \mathrm{~h}$, including two independent samples for $12 \mathrm{~h}$. Paired-end and strand-specific RNA-seq libraries were prepared according to Illumina instructions and sequenced on HiSeq 2000 (Illumina) with sequence length of 150 bp. Raw sequencing data were aligned to the human genome (hg19) using Tophat mapper, and transcripts were reconstructed using Cufflinks 2.02 (ref. 27). Annotation of the gene loci was performed using Gencode v19 as reference. Differential expression of the transcripts in response to treatment was assessed using Cuffdiff2 (ref. 23).

p53 chromatin immunoprecipitation. A total of $10^{7}$ HCT116 p53 $+/+$ cells were untreated or treated with 5 -FU for $12 \mathrm{~h}$ and crosslinked with formaldehyde (10 min $1 \%)$. Glycine was then added for $5 \mathrm{~min}$ to a final concentration of $0.125 \mathrm{M}$, cells scrapped and collected. The cell pellet was resuspended in lysis buffer $(5 \mathrm{mM}$ Tris- $\mathrm{HCl}, \mathrm{pH} 8.0,85 \mathrm{mM} \mathrm{KCl}, 0.5 \%$ NP40 supplemented with protease inhibitors cocktail from Roche) and the nuclear pellet was recovered by centrifugation and resuspended in RIPA buffer $(20 \mathrm{mM}$ Tris- $\mathrm{HCl}, \mathrm{pH} 7.5,100 \mathrm{mM} \mathrm{NaCl}, 1 \mathrm{mM}$ EDTA, $0.5 \%$ NP40, $0.5 \%$ Na-deoxycholate, $0.1 \%$ SDS supplemented with protease inhibitors cocktail). Nuclear extracts were sonicated using Bioruptor U-200.

The chromatin was precleared by adding dynabeads for $30 \mathrm{~min}$ at $4{ }^{\circ} \mathrm{C}$ and incubated overnight with p53 polyclonal antibody FL393 (Santa Cruz SC-6243). A total of $3 \mu \mathrm{g}$ of dynabeads was added for $1 \mathrm{~h}$ at $4^{\circ} \mathrm{C}$. The antibody was coupled with dynabeads followed by four washes with $\mathrm{LiCl}$ wash buffer $(100 \mathrm{mM}$ Tris, $\mathrm{pH} 7.5,500 \mathrm{mM} \mathrm{LiCl}, 1 \% \mathrm{NP} 40,1 \% \mathrm{Na}$-deoxycholate) and $1 \times$ with TE $(10 \mathrm{mM}$ Tris- $\mathrm{HCl}, \mathrm{pH} 7.5,1 \mathrm{mM}$ EDTA, pH 8.0). DNA was eluted for $1 \mathrm{~h}$ at $37^{\circ} \mathrm{C}$ in Elution Buffer ( $1 \% \mathrm{SDS}, 0.1 \mathrm{M} \mathrm{NaHCO}_{3}$ ), reverse X linked, purified and analysed by $\mathrm{qPCR}$ or used for sequencing library preparation.

p53 motif analysis. Genes were differentially selected for the de novo motif search performed with MEME-ChIP ${ }^{40}$ using the binding site sequences corresponding to these enriched regions with default parameters. TOMTOM tool of MEME-ChIP was used for the comparison of obtained DNA motifs against JASPAR database of known motifs.

Microarray analysis. For gene expression profiling, total RNA was extracted and hybridized to Affymetrix Human Transcriptome Array 2.0. Background correction and normalization were done using RMA (Robust Multichip Average) algorithm ${ }^{41}$ using Affymetrix Power Tools. After quality assessment, a filtering process was performed to eliminate low-expression probe sets. Applying the criterion of an expression value greater than 16 in two samples for each experimental condition, 41,697 probe sets were selected for statistical analysis. $\mathrm{R}$ and Bioconductor were used for preprocessing and statistical analysis. LIMMA (Linear Models for Microarray Data $)^{42}$ was used to find out the probe sets that showed significant differential expression between experimental conditions. Genes were selected as significant using a B-statistic cut-off $B>0$.

Functional enrichment analysis of Gene Ontology (GO) categories was carried out using standard hypergeometric test ${ }^{43}$. The biological knowledge extraction was complemented through the use of Ingenuity Pathway Analysis (Ingenuity Systems; www.ingenuity.com).

qPCR primers and ASOs. The qPCR primers and ASO sequences used in this study are listed in Supplementary Table 9.

All ASOs were designed and provided by ISIS Pharmaceuticals. All were $20 \mathrm{nt}$ in length and chemically modified with phosphorothioate in the backbone, five $2^{\prime}$-O-methoxyethyl residues at each terminus and a central deoxynucleotide region of 10 residues (5-10-5 gapmer). ASOs were synthesized using an Applied Biosystems 380B automated DNA synthesizer (PerkinElmer Life and Analytical Sciences-Applied Biosystems).

Cell proliferation assays. For proliferation analysis, 1,000 cells were plated per well in 96-well plates and assessed with a CellTiter96 Aqueous Non-Radioactive Cell Proliferation Assay (MTS) Kit G3581 (Promega).

Apoptosis and cell cycle analysis. At $24 \mathrm{~h}$ after transfection, $1 \times 10^{5}$ cells were plated in 96-well white microplates and treated for $24 \mathrm{~h}$ with $385 \mu \mathrm{M} 5$-FU. Apoptosis was determined by quantification with caspase-Glo 3/7 reagent (Promega) using a FLUOstar Optima luminometer, and with annexin V fluorescence-activated cell sorting using an Apoptosis Detection Kit I (cat-559763; BD Biosciences).

For cell cycle analysis, cells were labelled with propidium iodide (PI) and measured in the FACSCalibur flow cytometer (BD Biosciences). Data represent the mean \pm s.d. of a minimum of three biological replicates.

Human samples. Human colorectal carcinoma samples were provided by the Basque Biobank for Research-OEHUN (http://www.biobancovasco.org). Samples were processed following standard operation procedures with appropriate ethical approval by the Ethics Committee for Clinical Research of Donostia Hospital. An informed consent was obtained from all subjects. RNA was extracted with RNEASY MINI KIT (Qiagen) and reverse transcription was performed using random priming and Superscript Reverse Transcriptase (Life Technologies), according to the manufacturer's guidelines.

The $\triangle C_{\mathrm{t}}$ value was determined by subtracting the GAPDH $C_{\mathrm{t}}$ value from the p53-regulated lncRNAs' $C_{\mathrm{t}}$ value.

The performance as biomarker of each lncRNA was evaluated using ROC analysis ${ }^{44}$.

An algorithm based on logistic regression was applied to the obtained 5 -lncRNA-signature classification ${ }^{45}$.

Subcellular fractionation. A total of $10^{7}$ cells were trypsinized and washed once with cold PBS, aliquoted in two tubes and collected by centrifugation at $1,000 \mathrm{~g}$ for $5 \mathrm{~min}$ at $4{ }^{\circ} \mathrm{C}$. One cell pellet represented the whole-cell extract, while the other one was processed for the remaining subcellular fractions. Both pellets were resuspended in $500 \mu \mathrm{l}$ of Buffer A (10 mM Tris- $\mathrm{HCl}, \mathrm{pH} 7.5,1.5 \mathrm{mM} \mathrm{MgCl}_{2}, 140 \mathrm{mM}$ $\mathrm{NaCl}, 0.05$ IGEPAL supplemented with protease inhibitor cocktail and SuperaseIN $10 \mathrm{U} \mathrm{ml}^{-1}$ ), incubated for $10 \mathrm{~min}$ on ice and kept for subsequent RNA extraction. A total of $500 \mu \mathrm{l}$ of Buffer A plus sucrose $(10 \mathrm{mM}$ Tris- $\mathrm{HCl}, \mathrm{pH} 7.5,1.5 \mathrm{mM}$ $\mathrm{MgCl} 2,140 \mathrm{mM} \mathrm{NaCl} 0.05 \%$ IGEPAL, 50\% Sucrose) was added to the bottom of a clean eppendorf tube and the upper phase (whole-cell extract resuspended in Buffer A) was gently added to this tube preventing the mix of the two phases and centrifuged for $10 \mathrm{~min}$ at $4{ }^{\circ} \mathrm{C}$ and $12,000 \mathrm{~g}$ to obtain nuclear and cytoplasmic fractions. Around $500 \mu \mathrm{l}$ of the upper phase (cytoplasmic fraction) was collected and the rest was discarded, leaving the pellet (nuclear fraction). Total nuclear fraction was resuspended in $500 \mu \mathrm{l}$ of Buffer B $(10 \mathrm{mM}$ Tris, $100 \mathrm{mM} \mathrm{NaCl}, 1 \mathrm{mM}$ EGTA, $300 \mathrm{mM}$ sucrose, $0.5 \mathrm{mM} \mathrm{NaVO}$, $50 \mathrm{mM} \mathrm{NaF}, 1 \mathrm{mM}$ phenylmethylsulphonyl fluoride, $0.5 \%$ triton X-100, protease inhibitor cocktail and SuperasIN) and incubated for $10 \mathrm{~min}$ on ice to permeabilize the cells. To separate nuclear soluble from nuclear insoluble fraction, sample was centrifuged at $2,000 \mathrm{~g}$ for $5 \mathrm{~min}$ at $4{ }^{\circ} \mathrm{C}$ and the supernantant (nuclear soluble fraction) and pellet (nuclear insoluble/ chromatin fraction) were collected. The nuclear insoluble fraction was resuspended in Buffer A and finally $1 \mathrm{ml}$ of Trizol was added to all tubes for subsequent RNA extraction.

RNA fluorescence in situ hybridization. RNA fluorescence in situ hybridization for PR-lncRNA-1 and PR-lncRNA-10 detection was performed using a pool of 48 fluorescent probes purchased from Stellaris Biosearch Technologies, following the manufacturer's instructions.

Statistical analysis. Experimental data are represented as the mean \pm s.d. of a minimum of three biological replicates and were compared using Student's $t$-test. 
Significant $P$ values are indicated with asterisks as follows: ${ }^{*} P<0.05,{ }^{* *} P<0.01$ and ${ }_{* * * P}<0.001$

\section{References}

1. Beckerman, R. \& Prives, C. Transcriptional regulation by p53. Cold Spring Harb. Perspect. Biol. 2, a000935 (2010).

2. Menendez, D., Inga, A. \& Resnick, M. A. The expanding universe of $\mathrm{p} 53$ targets. Nat. Rev. Cancer 9, 724-737 (2009).

3. Dinger, M. E. et al. Long noncoding RNAs in mouse embryonic stem cell pluripotency and differentiation. Genome Res. 18, 1433-1445 (2008).

4. Khalil, A. M. et al. Many human large intergenic noncoding RNAs associate with chromatin-modifying complexes and affect gene expression. Proc. Natl Acad. Sci. USA 106, 11667-11672 (2009).

5. Cabili, M. N. et al. Integrative annotation of human large intergenic noncoding RNAs reveals global properties and specific subclasses. Genes Dev. 25, 1915-1927 (2011).

6. Guttman, M. et al. Chromatin signature reveals over a thousand highly conserved large non-coding RNAs in mammals. Nature 458, 223-227 (2009).

7. Ulitsky, I. \& Bartel, D. P. lincRNAs: genomics, evolution, and mechanisms. Cell 154, 26-46 (2013).

8. Rinn, J. L. et al. Functional demarcation of active and silent chromatin domains in human HOX loci by noncoding RNAs. Cell 129, 1311-1323 (2007).

9. Wang, K. C. \& Chang, H. Y. Molecular mechanisms of long noncoding RNAs. Mol. Cell 43, 904-914 (2011).

10. Rinn, J. L. \& Chang, H. Y. Genome regulation by long noncoding RNAs. Annu. Rev. Biochem. 81, 145-166 (2012).

11. Engreitz, J. M. et al. The Xist lncRNA exploits three-dimensional genome architecture to spread across the X chromosome. Science 341, 1237973 (2013).

12. Fatica, A. \& Bozzoni, I. Long non-coding RNAs: new players in cell differentiation and development. Nat. Rev. Genet. 15, 7-21 (2014).

13. Gupta, R. A. et al. Long non-coding RNA HOTAIR reprograms chromatin state to promote cancer metastasis. Nature 464, 1071-1076 (2010).

14. Yuan, J. H. et al. A long noncoding RNA activated by TGF-beta promotes the invasion-metastasis cascade in hepatocellular carcinoma. Cancer Cell 25, 666-681 (2014).

15. Li, L. et al. Role of human noncoding RNAs in the control of tumorigenesis. Proc. Natl Acad. Sci. USA 106, 12956-12961 (2009).

16. Huarte, M. et al. A large intergenic noncoding RNA induced by p53 mediates global gene repression in the p53 response. Cell 142, 409-419 (2010).

17. Marin-Bejar, O. et al. Pint lincRNA connects the p53 pathway with epigenetic silencing by the Polycomb repressive complex 2. Genome Biol. 14, R104 (2013).

18. Hung, T. et al. Extensive and coordinated transcription of noncoding RNAs within cell-cycle promoters. Nat. Genet. 43, 621-629 (2011).

19. Zhang, A. et al. The human long non-coding RNA-RoR is a p53 repressor in response to DNA damage. Cell Res. 23, 340-350 (2013).

20. Liu, Q. et al. LncRNA loc285194 is a p53-regulated tumor suppressor. Nucleic Acids Res. 41, 4976-4987 (2013).

21. Zhang, A., Xu, M. \& Mo, Y. Y. Role of the lncRNA-p53 regulatory network in cancer. J. Mol. Cell Biol. 6, 181-191 (2014).

22. Kaeser, M. D., Pebernard, S. \& Iggo, R. D. Regulation of p53 stability and function in HCT116 colon cancer cells. J. Biol. Chem. 279, 7598-7605 (2004).

23. Trapnell, C. et al. Differential gene and transcript expression analysis of RNA-seq experiments with TopHat and Cufflinks. Nat. Protoc. 7, 562-578 (2012).

24. Harrow, J. et al. GENCODE: the reference human genome annotation for The ENCODE Project. Genome Res. 22, 1760-1774 (2012).

25. Djebali, S. et al. Landscape of transcription in human cells. Nature $\mathbf{4 8 9}$, 101-108 (2012)

26. ENCODE Project Consortium et al. An integrated encyclopedia of DNA elements in the human genome. Nature 489, 57-74 (2012).

27. Trapnell, C. et al. Differential analysis of gene regulation at transcript resolution with RNA-seq. Nat. Biotechnol. 31, 46-53 (2013).

28. Wang, L. et al. CPAT: Coding-Potential Assessment Tool using an alignmentfree logistic regression model. Nucleic Acids Res. 41, e74 (2013).

29. Kenzelmann Broz, D. et al. Global genomic profiling reveals an extensive p53-regulated autophagy program contributing to key p53 responses. Genes Dev. 27, 1016-1031 (2013).

30. Bode, A. M. \& Dong, Z. Post-translational modification of $\mathrm{p} 53$ in tumorigenesis. Nat. Rev. Cancer 4, 793-805 (2004).
31. Schwartz, D. \& Rotter, V. P53-dependent cell cycle control: response to genotoxic stress. Semin. Cancer Biol. 8, 325-336 (1998).

32. Cancer Genome Atlas Network. Comprehensive molecular characterization of human colon and rectal cancer. Nature 487, 330-337 (2012).

33. Wood, L. D. et al. The genomic landscapes of human breast and colorectal cancers. Science 318, 1108-1113 (2007).

34. Vogelstein, B. et al. Genetic alterations during colorectal-tumor development. New Engl. J. Med. 319, 525-532 (1988).

35. Fearon, E. R. \& Vogelstein, B. A genetic model for colorectal tumorigenesis. Cell 61, 759-767 (1990)

36. Idogawa, M. et al. Identification and analysis of large intergenic non-coding RNAs regulated by p53 family members through a genome-wide analysis of p53-binding sites. Hum. Mol. Genet. 23, 2847-2857 (2014).

37. Toledo, F. \& Wahl, G. M. Regulating the p53 pathway: in vitro hypotheses, in vivo veritas. Nat. Rev. Cancer 6, 909-923 (2006).

38. Marques, A. C. \& Ponting, C. P. Intergenic lncRNAs and the evolution of gene expression. Curr. Opin. Genet. Dev. 27C, 48-53 (2014).

39. Rodrigues, N. R. et al. P53 mutations in colorectal cancer. Proc. Natl Acad. Sci. USA 87, 7555-7559 (1990).

40. Ma, W., Noble, W. S. \& Bailey, T. L. Motif-based analysis of large nucleotide data sets using MEME-ChIP. Nat. Protoc. 9, 1428-1450 (2014).

41. Irizarry, R. A. et al. Summaries of Affymetrix GeneChip probe level data. Nucleic Acids Res. 31, e15 (2003).

42. Smyth, G. K. Linear models and empirical bayes methods for assessing differential expression in microarray experiments. Stat. Appl. Genet. Mol. Biol. 3 Article3 (2004).

43. Draghici, S. Data Analysis Tools For DNA Microarrays (Chapman \& Hall/CRC, 2003).

44. Sing, T., Sander, O., Beerenwinkel, N. \& Lengauer, T. ROCR: visualizing classifier performance in R. Bioinformatics 21, 3940-3941 (2005)

45. Hornik, K., Buchta, C. \& Zeileis, A. Open-Source machine Learning: R meets weka. Comput. Stat. 24, 225-232 (2009).

\section{Acknowledgements}

Our research was supported by European Research Council Starting Grant 281877 and Spanish Ministry of Science Grants BFU2011-23485 and SRYC1100I008347XV0. We thank The ENCODE project and Gene Expression Omnibus (GEO) database for providing their platforms and contributors for their valuable data sets. We also thank Lidia López for technical assistance and the Basque Biobank for providing the human samples used in this study.

\section{Author contributions}

M.H. and Y.S. designed the research; Y.S., F.P.M., O.M.-B. and J.G. performed the research; V.S. and A.A. performed the data analysis; A.M. and L.B. contributed to the collection of patient samples; S.G. contributed with design, testing and production of reagents; and M.H. and Y.S. wrote the paper.

\section{Additional information}

Accession codes: Primary data from RNA-seq, p53 ChIP-seq and microarray analyses have been deposited in the Gene Expression Omnibus under the accession code GSE58528.

Supplementary Information accompanies this paper at http://www.nature.com/ naturecommunications

Competing financial interests: The authors declare no competing financial interests.

Reprints and permission information is available online at http://npg.nature.com/ reprintsandpermissions/

How to cite this article: Sánchez, Y. et al. Genome-wide analysis of the human p53 transcriptional network unveils a lncRNA tumour suppressor signature. Nat. Commun. 5:5812 doi: 10.1038/ncomms6812 (2014).

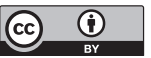

This work is licensed under a Creative Commons Attribution 4.0 International License. The images or other third party material in this article are included in the article's Creative Commons license, unless indicated otherwise in the credit line; if the material is not included under the Creative Commons license, users will need to obtain permission from the license holder to reproduce the material. To view a copy of this license, visit http://creativecommons.org/licenses/by/4.0/ 